\title{
Discrepancies of HIV-1 Reverse Transcriptase Resistance Interpretation of Insertions and Deletions between Two Genotypic Algorithms
}

\author{
Giselle Ibette Silva López-Lopes André Minhoto Lança \\ João Leandro de Paula Ferreira Luciana Oliveira Souza \\ Luís Fernando de Macedo Brígido \\ Laboratório de Retrovírus, Centro de Virologia, Instituto Adolfo Lutz, São Paulo, Brazil
}

\section{Key Words}

Highly active antiretroviral therapy · HIV-1 antiretroviral drugs · HIV Stanford Resistance Database .

Geno2Pheno ${ }_{\text {[resistance] }}$

\begin{abstract}
Background: Bioinformatics algorithms have been developed for the interpretation of resistance from sequence submission, which supports clinical decision making. This study evaluated divergences of the interpretation of the genotyping in two commonly used algorithms, using sequences with indels of reverse transcriptase genes. Methods: Sequences were obtained from virus RNA of patients failing highly active antiretroviral therapy from 2004 to 2011. Alignments were obtained using Clustal W including subtype B consensus and HXB2. Sequences with evidence of indels were submitted to the Stanford Resistance Database and to the Geno2Pheno to locate indel positioning and determine the resistance profile. Results: A total of 1,959 partial reverse transcriptase sequences were assessed, mostly subtype B (74\%). Insertions and deletions were observed in 0.9 and $0.6 \%$ of sequences, respectively. Discordant insert positioning was assigned for most (90\%) insertion sequences, with $27 \%$ discordances for deletions. Susceptibility differed for some antiretroviral
\end{abstract}

\section{KARGER}

E-Mail karger@karger.com

www.karger.com/int drugs, predominantly for TDF, d4T and ETV, when sequences with deletions were evaluated. Conclusion: Both indel positioning and its impact on drug susceptibility varies depending on the algorithm, a fact that might influence the clinical decision. Critical analysis of indel sequences with manual alignments is important, and its use alongside different algorithms may be important to better understand the outcomes of genotypic resistance prediction.

Copyright $\odot 2013$ S. Karger AG, Base

\section{Introduction}

The HIV-1 polymerase gene is responsible for coding different enzymes which are essential for the retrovirus replicative cycle, including the protease and reverse transcriptase (RT) that are targets for most of HIV-1 antiretroviral (ARV) drugs. During reverse transcription, a lack of the proofreading properties of the enzyme, known for its relatively high error rate, is one of the factors responsible for viral diversity, an exquisite environment for the emergence of mutant viral strains. Also, the selective ef-

G.I.S. López-Lopes and A.M. Lança contributed equally to this work.
(C) 2013 S. Karger AG, Basel

0300-5526/13/0564-0217\$38.00/0
Dr. Luís Fernando de Macedo Brígido, MD PhD

Dr. Arnaldo Av., 355

Cerqueira Cesar

São Paulo 01246-902 (Brazil)

E-Mail lubrigido@gmail.com 
fect of the several ARV drugs composing the armamentarium of HIV-1 therapy on viral replication is essential to the development of the resistance observed in treatment failure [1]. As mutations can be transmitted [2], its impact on treatment options, both for initial and salvage therapies, constitutes an important public health issue. Over two hundred mutations have been associated with drug resistance, with complex mutation patterns in patients on highly active antiretroviral therapy (HAART) [3]. The evaluation of susceptibility to ARV drugs is of great importance in providing alternatives to salvage therapy in patients failing treatment, and genotyping tests have been employed to identify mutations that may confer resistance to ARV drugs [4]. The majority of substitutions confer cross-resistance drugs of the same class [5], such as K65R that reduces susceptibility to almost all nucleotide analogue reverse transcriptase inhibitors (NRTI) [6]. Moreover, strains exhibiting insertions of multiple nucleotides in protease and RT genes have been found in patients who have failed ARV therapy, and compensatory mutations are favored by drug-selective pressure on resistant strains replicating in a viremic patient [7]. Among the various mutations that confer reduced susceptibility, insertions and deletions, often called indels, can be observed in $0.5-2.4 \%$ of heavily treated patients $[8,9]$.

Along with sequence data per se, different interpretation algorithms have been developed to allow fast and concise resistance profile definition from sequence submission. Some of the genotyping algorithms are freely available, namely the HIV Stanford Resistance Database (HIVdb) [5] and the Geno2Pheno ${ }_{\text {[resistance] }}(\mathrm{G} 2 \mathrm{P})$ [10]; these are two of the algorithms most commonly used by researchers and physicians worldwide. However, while most of the time these various systems agree, the interpretation of some sequences can be rather divergent. Several authors have made use of different criteria to establish a comparison between those different methods of resistance prediction [11-18]. However, the literature lacks a comparison of algorithms regarding when insertions or deletions of amino acids are present and how the positioning of these codons in the websites contributes to different susceptibility predictions. The aim of this study was to evaluate the divergences in the interpretation of the genotyping in two different algorithms using a routine resistance sequence dataset with insertions and deletions in the RT gene. Also, the association of the indels with other mutations was evaluated to better understand their role in patients extensively exposed to HAART.

\section{Patients and Methods}

\section{Sequence Selection}

Sequences were obtained from plasma RNA of patients failing HAART, with a viral load over 2,000 copies/ml $\left(3.3 \log _{10}\right)$, while they were attending facilities administered by the public health system of São Paulo and nearby cities, as part of routine resistance genotyping from 2004 and 2011. HIV-1 RT gene sequences were obtained using ViroSeq (Abbott Laboratories, USA) resolved on ABI 3100 Genetic Analyzer (until 2008) or TRUGENE ${ }^{\circledR}$ HIV-1 Genotyping Kit for Drug Resistance resolved on OpenGene (Siemens Healthcare Diagnostics, USA) thereafter. All sequences obtained were screened for the presence of insertions and deletions.

Multiple sequence alignments were obtained using the Clustal $\mathrm{W}$ algorithm embedded in BioEdit software including subtype $\mathrm{B}$ consensus and HXB2 reference sequences downloaded from Los Alamos National Laboratory (LANL) database (http://www.hiv. lanl.gov/content/sequence/HIV/mainpage.html) [19].

\section{Sequence Submission and Indel Positioning}

Sequences with evidence of indels were submitted to HIVdb version 6.2.0 (last update May 29, 2012) and G2P version 3.3 (last update September 13, 2012) to detect and locate insertion/deletion positioning, and to determine the drug susceptibility profile. Manual entering of individual mutations in HIVdb was also evaluated to verify if this would change the prediction made by HIVdb automatic submission. Moreover, a manual alignment was conducted to allow indel positioning. The determination of which would be the most probable positioning of the indels was based on the prior conservative assumption that deletions at position 67 and insertions at codon 69 are the most common changes in this genomic region associated with genetic resistance to ARV drugs [20, 21]. The amino acid composition of all sequences was evaluated to document the prevalence of resistance mutations in both groups.

\section{Interpretation of Genotyping}

$\mathrm{HIVdb}$ stratifies resistance in five levels $(\mathrm{H}=$ high-level resistance, $\mathrm{I}=$ intermediate resistance, $\mathrm{L}=$ low-level resistance, $\mathrm{P}=$ potential low-level resistance and $S=$ susceptible), while G2P stratifies it in three levels $(1=$ resistant, $2=$ intermediate and $3=$ susceptible). Our paired analysis collapsed the scores 'potential low-level resistance' as 'susceptible' and the low level as 'intermediate resistance' ( $1=\mathrm{S}, \mathrm{P}, \mathrm{L} ; 2=\mathrm{L}, \mathrm{I} ; 3=\mathrm{H})$, as suggested by the $\mathrm{HIVdb}$ HIValg program. Differences between algorithms were evaluated as partial discordance, when the algorithms disagree at the level of resistance or between susceptible and intermediate, or as full discordance, when one algorithm scores a sequence as susceptible and the other shows a fully resistant profile. The International AIDS Society mutation list update from 2011 [5] was also used in the characterization of mutations associated with resistance.

\section{Results}

A total of 2,272 RT partial gene sequences were screened and those with available data such as age, gender, CD4, viral load and treatment regimens were selected. Also, only the first sequences from each patient were 
Fig. 1. Deduced amino acids of the indel regions with positioning according to the manual alignment, to G2P and to HIVdb. Hyphens represent gaps in the alignment, while black boxes highlight the different G2P and HIVdb positioning of indels. Codon numbering is adjusted to the HXB2 reference. Samples BR185SP11* and BR577SP09* are subsequent samples of patients BR085SP10 and BR067SP07, respectively.

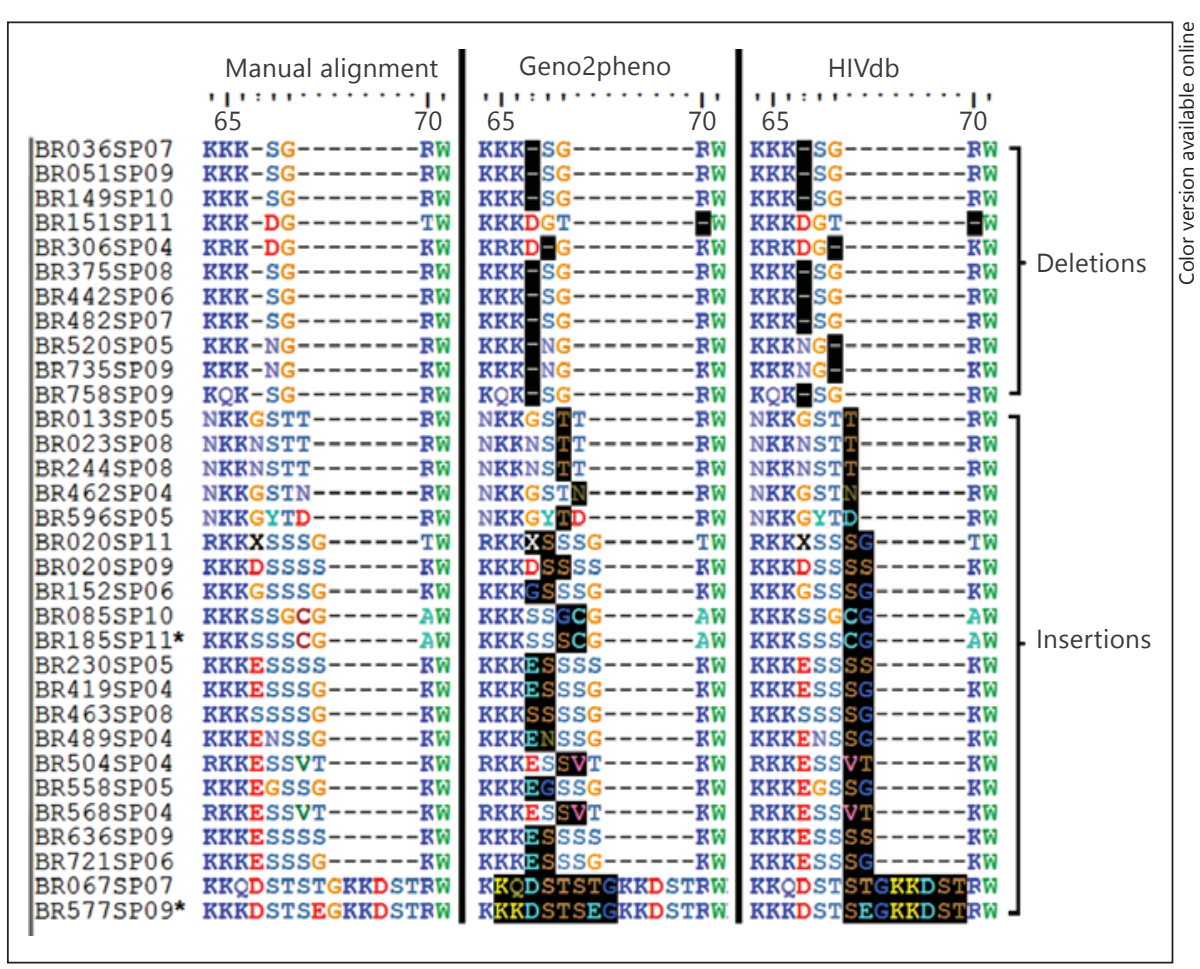

included, i.e. a total of 1,959 sequences. Thirty (1.4\%) of these were found to contain amino acid insertions (0.9\%) or deletions (0.6\%) (fig. 1). Patients' characteristics according to the indel type as well as from the remaining 1,929 of nonindel cases are detailed in table 1 .

\section{Sequence Analysis}

Nineteen sequences exhibited an insertion of 1,2 or 8 amino acids at codon 69 according to manual alignment (fig. 1). Thirteen presented the usual insertion of 2 amino acids $\left(69\right.$ ins $\left.^{2}\right)$ followed by T69S substitution in most of those sequences $(92.3 \%)$. The insertions observed were seven SG (53\%), three SS (23.6\%), two VT (15.4\%) and one CG $(7.7 \%)$. Five sequences showed the presence of 1 -amino acid insertion (69ins $\left.{ }^{1}\right)$, mostly $\mathrm{T}(60 \%)$, and all those sequences exhibited the substitution K64N. Additionally, one sequence showed an unusual 8-amino acid insertion $\left(69 \mathrm{ins}^{8}\right)$. Along the insertion sequences eleven isolates had deletions at codon $67(\Delta 67)$; the substitution T69G was observed in all strains and the mutations $568 \mathrm{~N}$ or S68D in $16.2 \%$ each.

Sequences were also evaluated for resistance profiles according to the 2011 IAS [5] resistance mutation list grouped by $\Delta 67,69$ ins $^{1}, 69$ ins $^{2}$ and 69 ins $^{8}$. Within the 69 ins $^{2}$ group, the prevalent resistance mutations were the thymidine analogue mutations type I (TAM-I), as well as
A62V and M184 V/I. On the other hand, along the 69ins ${ }^{1}$, the most common resistance mutations were the TAM type II (TAM-II) followed by T215F. The third group, $\Delta 67$, had a high prevalence of both TAM-I and TAM-II, as well as L74I and M184V. The single 69ins ${ }^{8}$ sequence showed the TAM-I substitutions M41L, L210W and T215F, as well as the TAM-II K70R. Data of the NRTI mutations are detailed in table 2.

\section{Comparison of Algorithms}

$\mathrm{HIVdb}$ and G2P alignment algorithms were compared both as positioning concordance as well as indel impact on drug susceptibility. Regarding positioning, the insertions were discordant for $90 \%$ of sequences, while the discordance was $27 \%$ in samples with deletions. All 19 events of insertion were positioned at codon 69 by HIVdb, while positioning varied between 64 and 69 when using G2P. Sequences defined by HIV db as 69SG/SS were alternatively classified as $66 \mathrm{ins}^{2}$ in G2P, with the exception of one 69SS sequence defined as 67ins ${ }^{2}$. Those containing 69CG/ VT had its positioning set as 68 ins $^{2}$ in G2P. No difference was observed among the $69 \mathrm{D}$ and $69 \mathrm{~N}$ sequences, both being classified as 69 ins $^{1}$. The three $69 \mathrm{~T}$ insertions were positioned at 68 by G2P. Furthermore, the sequence containing the 8 -amino acid insertion was positioned at codon 64 . The patient with this variant (BR067SP07) had another 
Table 1. Clinical and demographic characteristics of the study patients according to indel type

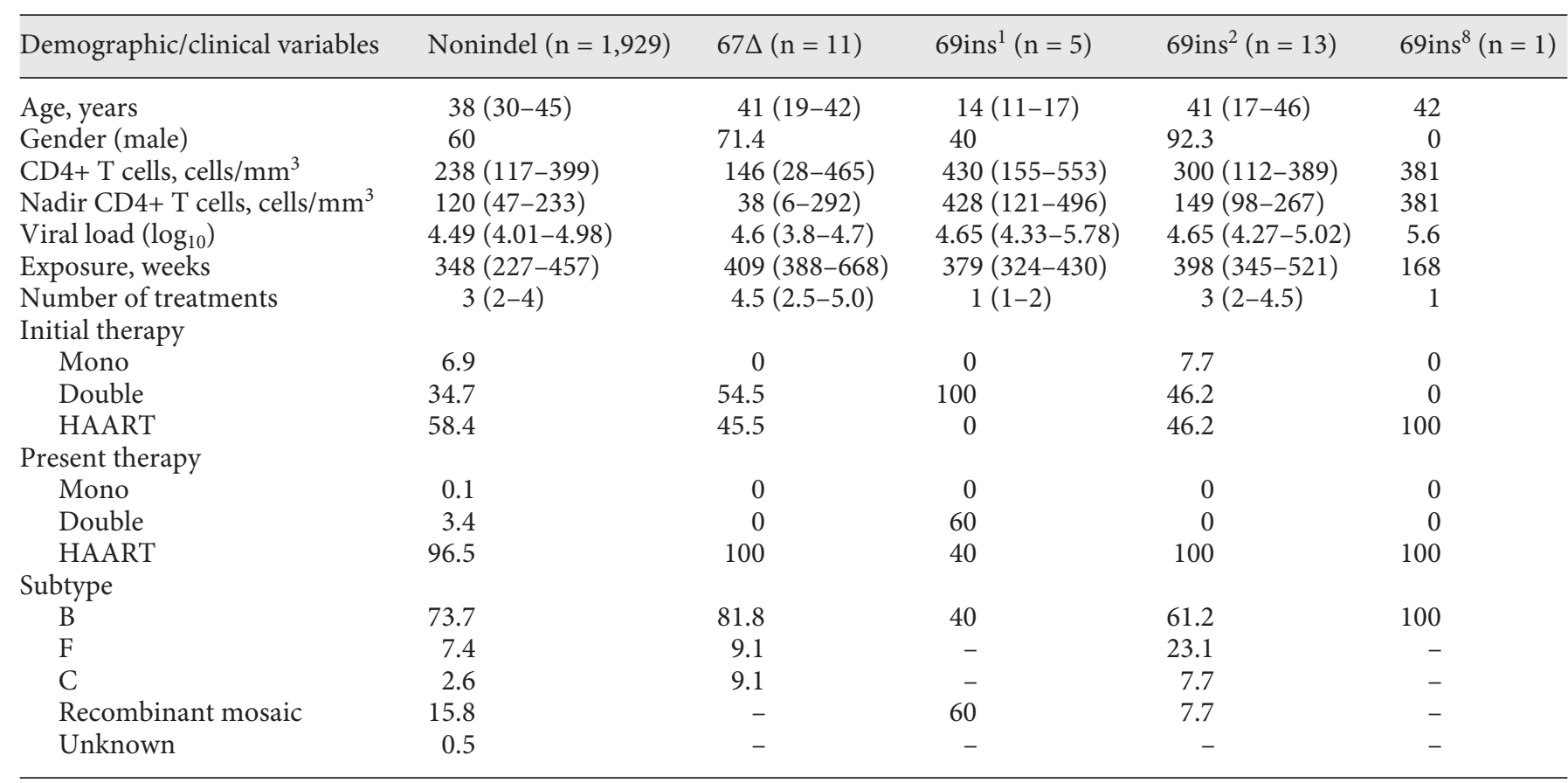

Results are expressed as the median with the interquartile range in parentheses or as percentage. Nonindels $=$ From sequences without indels at RT; $\Delta 67=$ deletion at codon $67 ; 69$ ins $^{1}, 69$ ins $^{2}$ and 69 ins $^{8}=$ insertion of 1,2 and 8 amino acids at codon 69 , respectively.

Table 2. Resistance mutations associated with NRTIs in the different groups of indels according to the 'IAS Resistance Update 2011' [5]

\begin{tabular}{|c|c|c|c|c|c|c|c|c|c|c|c|c|c|c|c|c|}
\hline & $\mathrm{n}$ & M41L & $\mathrm{A} 62 \mathrm{~V}$ & K65R & $D 67 N$ & K70R & L74I/V & V75I/M & F77L & Y115F & F116Y & Q151M & M184I/V & L210W & $\mathrm{T} 215 \mathrm{~F} / \mathrm{Y}$ & K219E/Q \\
\hline Control & 1,929 & 33 & 4 & 2 & 30 & 20 & $4 / 4$ & $2 / 7$ & 3 & 1 & 2 & 2 & $1 / 62$ & 24 & $12 / 37$ & \\
\hline$\Delta 67$ & 11 & 36 & & 9 & & 73 & $45 / 0$ & & 9 & & 9 & 9 & $0 / 91$ & 18 & $46 / 36$ & $80 / 0$ \\
\hline 69 ins $^{2}$ & 13 & 69 & 46 & & & & $12 / 12$ & $0 / 31$ & 15 & & & & $23 / 54$ & 54 & $17 / 75$ & $0 / 8$ \\
\hline 69 ins $^{8}$ & 1 & 100 & & & & 100 & & & & & & & & 100 & $100 / 0$ & \\
\hline
\end{tabular}

Results are expressed as percentage. TAM-I are given in bold, while TAM-II are given in italics.

genotype test performed 2 years later that showed the persistence of this insertion, as well one other case with subsequent sample evaluation; these are included in figure 1. Regarding the deletions, all 7 sequences exhibiting the wild-type S68 (according to manual alignment) were classified as $\Delta 67$ in both HIVdb and G2P, while the two S68N mutations had their deletion positioned at codon 69 by HIVdb and 67 by G2P. Moreover, the remaining two sequences exhibiting S68D were classified as $\Delta 70$ by G2P, while HIVdb positioned the deletion at 68 and 69 codons (fig. 1). The manual entering of mutations either by typing or by using the drop-down menu offered as HIVdb Mutation List option did not differ from the susceptibility results at automatic sequence analysis. However, some differences were seen at positioning, when the drop-down menu was used, where some uncommon changes exhibited at automatic analysis were not available for selection, e.g. BR11SP151's K70d (deletion). This could be solved by manually typing in those mutations. Both alternatives did not change the susceptibility profile.

The prediction of susceptibility to each drug of the two algorithms was evaluated separately for sequences har- 
boring insertions or deletions. The exhibited partial and full discordance between algorithms is detailed in table 3, with some discordance observed for all ARV drugs. Partial or full discordance was observed for a new generation of non-NRTI drugs, ETV (37\% for insertions and 73\% for deletions), as for NRTI drugs, especially $\mathrm{d} 4 \mathrm{~T}$ and TDF. Additionally, there was full discordance in $2(10 \%)$ insertion samples (ddI and ETR). Full discordance was more common among sequences with deletions (36\%). Using different combinations of HIV db classifications (e.g. considering low as susceptible or potential-low as intermediate at G2P) did not show important differences in algorithm comparisons. It is of note that all full discordant sequences were resistant at $\mathrm{HIVdb}$ and susceptible at G2P.

\section{Discussion}

Several computational algorithms of the interpretation of the genotyping of HIV-1 viral strains have been developed to better understand and characterize resistance in clinical samples. Alongside patients' treatment history, the genotypic algorithms may be employed to predict resistance to ARV drugs and help physicians with a fast and reliable method of determining the most adequate HAART option for each individualized salvage regimen. However, differences between the construction and the in vivo and in vitro resistance information used for each algorithm development may have an impact on phenotypic prediction and may lead to different resistance profiles. Two of the most frequently used algorithms available, HIV db and G2P, were selected for comparison. Our cohort includes mostly heavily treated patients, with a median of 7 years on ARV treatment, in many of whom treatment was initiated in the 1990s. From all sequences screened for indels, deletions (0.6\%) and insertions (0.9\%) among patients failing HAART were present in a prevalence that did not differ from the findings of Tamalet et al. [8], Winters and Merigan [7] and Harrigan et al. [9].

The indel groups, separated by the number of amino acids inserted, and the nonindel (control) group were compared. With exception of 69ins ${ }^{1}$, these groups were highly homogeneous regarding demographic, laboratory and treatment data (table 1). However, the 69ins ${ }^{1}$ group was mostly comprised of children $(80 \%$ under 18 years old), a population with immunological, virological and therapeutic peculiarities (e.g. ARV options available to children and adolescents were more limited at that time, and dual NRTI therapy was used for longer periods of time). Among insertion sequences, a high het-

Discrepancies in Interpretation of RT

HIV-1 Insertions and Deletions
Table 3. NRTI and NNRTI drugs susceptibility algorithms prediction agreement between Stanford HIVdb (version 6.2.0) and Geno2Pheno (version 3.3)

\begin{tabular}{|c|c|c|c|c|}
\hline \multirow[t]{3}{*}{ ARV } & \multicolumn{4}{|c|}{ Stanford HIVdb and geno2pheno discordances } \\
\hline & \multicolumn{2}{|c|}{ insertions $(n=19)$} & \multicolumn{2}{|c|}{ deletions $(n=11)$} \\
\hline & partial & full & partial & full \\
\hline ZDV & $0 \%$ & $0 \%$ & $18 \%$ & $0 \%$ \\
\hline ddI & $16 \%$ & $5 \%$ & $0 \%$ & $0 \%$ \\
\hline $\mathrm{d} 4 \mathrm{~T}$ & $37 \%$ & $0 \%$ & $36 \%$ & $27 \%$ \\
\hline 3TC & $32 \%$ & $0 \%$ & $9 \%$ & $0 \%$ \\
\hline $\mathrm{ABC}$ & $5 \%$ & $0 \%$ & $27 \%$ & $0 \%$ \\
\hline TDF & $26 \%$ & $0 \%$ & $73 \%$ & $0 \%$ \\
\hline NVP & $11 \%$ & $0 \%$ & $0 \%$ & $9 \%$ \\
\hline EFV & $21 \%$ & $0 \%$ & $36 \%$ & $9 \%$ \\
\hline ETR & $32 \%$ & $5 \%$ & $73 \%$ & $0 \%$ \\
\hline
\end{tabular}

Partial $=$ partial discordant; full $=$ full discordant. Partial discordance was considered when the level of resistance disagreed, as one algorithm classified as intermediate resistance, while the other classified as resistant or susceptible. Full discordant refers to full disagreement, as susceptible versus fully resistant.

erogeneity of both substitutions and insertions was observed, where 1, 2 or 8 amino acids were identified. Also, the genotypic resistances to NRTI differed among the sequences harboring one and two insertions. The resistance mutations exhibited by 69 ins $^{2}$ sequences were in agreement with the IAS 2011 mutation list regarding the 69 insertion complex [5]. Interestingly, recently HIVdb [22] has included in the 69 insertion comments as separate observations regarding 69 single and double inserts, where the former are often accompanied by TAM-II, while the latter are followed by TAM-I. Our data agree with HIVdb and suggest that a distinct mutation pattern may be associated with either a single or a double insertion, with potential impact on drug susceptibility. K64N, a rare mutation found by Rakik et al. [23] on a 69ins ${ }^{1}$ sequence, was also observed on all our sequences harboring single insertions. A distinct insertion of 8 amino acids in this same region $\left(69\right.$ ins $\left.^{8}\right)$ was detected in one case. A second sample, obtained 2 years after the first genotyping and a subsequent change of drug therapy, confirmed the presence of the long insertion, along some substitutions in the nearby positions (fig. 1). Large insertions between codons 64 and 70 are rather uncommon, although insertions/duplications of up to 15 amino acids have already been described $[9,24]$. Furthermore, a very similar substitution to the one observed in our patient was found by Van der Hoek et al. [25], who suggested 
that this large insertion would be the result of both the duplication of the virus nucleotides and the insertion of fifteen nucleotides from the human genomic DNA. No sequence of our patient's virus prior to HAART was available to investigate if the insertion emerged during or prior to therapy.

Susceptibility to ARV drugs is the most important aspect of resistance algorithm interpretation. To evaluate the impact of indels, both HIVdb and G2P were compared to identify interpretation mismatches for each different drug. We found a low algorithm prediction agreement for many drugs, both NRTI and non-NRTI in sequences harboring insertions, mainly to d4T, ETR, TDF and 3TC. An even higher degree of discordance was observed in $\Delta 67$ sequences for $\mathrm{d} 4 \mathrm{~T}, \mathrm{TDF}, \mathrm{EFV}$ and ETR. It is of note that a quarter of the $\Delta 67$ sequences were considered susceptible to $\mathrm{d} 4 \mathrm{~T}$ by G2P, while they were considered fully resistant according to HIVdb. The differences between indel positioning and susceptibility to ARV in both HIVdb and G2P reveal a lack of homogeneity in the way in which these algorithms handle the different indel strains. In fact, the HIV db algorithm considers the mutation individually and by default treats any insertion or deletion between positions 66 and 71 as $\Delta 67$ and 69ins, respectively, and the impact of each individual mutations is unclear. G2P, although identifies the indels, does not elucidate how each mutation is taken into account in determining the degree of resistance. We artificially changed the sequence by creating or removing an indel, and observed the way in which the algorithm interprets other mutations in the same sequence changes, such as T215Y/F, by scoring from polymorphism to resistance mutation (data not shown). These differences in the alignment and the interpretation of the genotyping of both algorithms can be fundamental in explaining why a high divergence between algorithms was observed for each drug. Therefore, in the absence of a phenotypic resistance test, a critical analysis of the sequence, along with the treatment history of the patient might be required for the proper interpretation of resistance prediction, especially in the presence of indels. The use of different algorithms simultaneously, along with a manual alignment of the sequences, might help identify what could be the most probable correct position of the change, and whether it corroborates or not with the automatic prediction available at those websites.

\section{Acknowledgments}

We would like to thank Fabio A.B. Cabral, Jaqueline S. Cavalcanti, Joao P.G. Batista, Antonio F.A.C. Siqueira, Karina P. Barroso and Mariucha C. Barbosa for part of sample analysis and the staff of the Retrovirus Laboratory, Adolfo Lutz Institute Sao Paulo, that have greatly improved this work. The Brazilian AIDS National Program provided the resistance tests. Funding was received from FAPESP 2006/61311-0 and 2011/21958-2.

\section{References}

1 Hirsch MS, Brun-Vezinet F, Clotet B, et al: Antiretroviral drug resistance testing in adults infected with human immunodeficiency virus type 1: recommendations of an International AIDS Society-USA Panel. Clin Infect Dis 2003;37:113-128.

2 Wainberg MA, Cameron DW: HIV resistance to antiviral drugs: public health implications. Drug Resistance Updates 1998;1:104-108.

3 Larder B, Wang D, Revell A: Application of artificial neural networks for decision support in medicine. HIV Resistance Response Database Initiative, London, UK. Methods Mol Biol 2008;58:123-136.

4 Hertogs K, de Béthune MP, Miller V, et al: A rapid method for simultaneous detection of phenotypic resistance to inhibitors of protease and reverse transcriptase in recombinant human immunodeficiency virus type 1 isolates from patients treated with antiretroviral drugs. Antimicrob Agents Chemother 1998;42:269-276.

5 Johnson VA, Calvez V, Günthard HF, et al: 2011 update of the drug resistance mutations in HIV-1. Top Antivir Med 2011;19:156-164.
6 6 Deval J, White KL, Miller MD, et al: Mechanistic basis for reduced viral and enzymatic fitness of HIV-1 reverse transcriptase containing both K65R and M184V mutations. J Biol Chem 2004;279:509-516.

7 Winters MA, Merigan TC: Insertions in the human immunodeficiency virus type 1 protease and reverse transcriptase genes: clinical impact and molecular mechanisms. Antimicrob Agents Chemother 2005;49:2575-2582.

$\checkmark 8$ Tamalet C, Yahi N, Tourrès C, et al: Multidrug resistance genotypes (insertions in the $\beta 3$ - $\beta 4$ finger subdomain and mdr mutations) of HIV-1 reverse transcriptase from extensively treated patients: incidence and association with other resistance mutations. Virology 2000;270:310-316.

-9 Harrigan PR, Mo T, Hirsch J, et al: A 21-base pair insertion/duplication at codon 69 of the HIV type 1 reverse transcriptase in a patient undergoing multiple nucleoside therapy. AIDS Res Hum Retroviruses 2007;24:895899.
10 Beerenwinkel N, Däumer M, Oett $M$, et al: Geno2pheno: estimating phenotyping drug resistance from HIV-1 genotypes. Nucleic Acids Res 2003;31:3850-3855.

-11 Stürmer M, Doerr HW, Staszewski S, et al: Comparison of nine resistance interpretation systems for HIV-1 genotyping. Antivir Ther 2003;8:239-244.

-12 De Luca A, Cingolani A, Di Giambenedetto, et al: Variable prediction of antiretroviral treatment outcome by different systems for interpreting genotypic human immunodeficiency virus type 1 drug resistance. J Infect Dis 2003; 187:1934-1943.

13 Ravela J, Betts BJ, Brun-Vezinet F, et al: HIV-1 protease and reverse transcriptase mutation patterns responsible for discordances between genotypic drug resistance interpretation algorithms. J Acquir Immune Defic Syndr 2003;33:8-14.

14 Kijak GH, Rubio A, Pampuro SE, et al: Discrepant results the interpretation of HIV-1 drug-resistance genotypic data among widely used algorithms. HIV Med 2003;4:72-78. 
15 Snoeck J, Kantor R, Shafer RW, et al: Discordances between interpretation algorithms for genotypic resistance to protease and reverse transcriptase inhibitors of human immunodeficiency virus are subtype dependent. Antimicrob Agents Chemother 2006;50:694-701.

16 Liu L, May S, Richman DD, et al: Comparison of algorithms that interpret genotypic HIV-1 drug resistance to determine the prevalence of transmitted drug resistance. AIDS 2008;22: 835-839.

17 Ntemgwa M, Gill MJ, Brenner BG, et al: Discrepancies in assignment of subtype/recombinant forms by genotyping programs for HIV type 1 drug resistance testing may falsely predict superinfection. AIDS Res Hum Retroviruses 2008;24:995-1002.

18 Kandathil AJ, Kannangai R, Cherian O, et al: A comparison of interpretation by three different HIV type 1 genotyping drug resistance algorithms using sequences from non-clade $B$ HIV type 1 strains. AIDS Res Hum Retroviruses 2009;25:315-318.
19 Sequence search interface. HIV sequence database. Los Alamos National Laboratory (last GenBank update: June 18, 2012; accessed: June 31, 2012). http://www.hiv.lanl.gov/con tent/sequence/HIV/mainpage.html.

20 Ross L, Johnson M, Ferri RG, et al: Deletions in the $\beta 3-\beta 4$ hairpin loop of HIV-1 reverse transcriptase are observed in HIV-1 isolated from subjects during long-term antiretroviral therapy. J Hum Virol 2000;3:144-149.

21 Imamichi T, Murphy MA, Imamichi H, Lane HC: Amino acid deletion at codon 67 and Thr-to-Gly change at codon 69 of human immunodeficiency virus type 1 reverse transcriptase confer novel drug resistance profiles. J Virol 2001;75:3988-3992.

22 HIVdb Program: Genotypic Resistance Interpretation Algorithm. HIV Drug Resistance Database. Stanford University (update: May 29, 2012; accessed: in June 31, 2012). http:// hivdb.stanford.edu.
23 Rakik AR, Ait-Khaled M, Griffin P, et al: A novel genotype encoding a single amino acid insertion and five other substitutions between residues 64 and 74 of the HIV-1 reverse transcriptase confers high-level cross-resistance to nucleoside reverse transcriptase inhibitors. Abacavir CNA2007 International Study Group. J Acquir Immune Deficit Syndr 1999;22: 132-139.

24 Lobato RL, Kim EY, Kagan RM, Merigan TC: Genotypic and phenotypic analysis of a novel 15-base insertion occurring between codons 69 and 70 of HIV type 1 reverse transcriptase. AIDS Res Hum Retroviruses 2002;18:733736.

25 Van der Hoek L, Back N, Jebbink MF, et al: Increase multinucleoside drug resistance and decreased replicative capacity of a human immunodeficiency virus type 1 variant with an 8-amino-acid insert in the reverse transcriptase. J Virol 2005;79:35363543. 